\title{
LINC complex independent perinuclear actin organization and cell migration
}

\author{
ANDREA FRACCHIA ${ }^{1,2}$; GABI GERLITZ ${ }^{1, *}$ \\ ${ }^{1}$ Department of Molecular Biology, Faculty of Life Sciences and Ariel Center for Applied Cancer Research, Ariel University, Ariel, 40700, Israel \\ 2 Department of Bone Marrow Transplantation and Cancer Immunotherapy, Hadassah Medical Organization, Jerusalem, 91120, Israel
}

Key words: Cell migration, Actin, Lamins, Nuclear envelope, Cancer

\begin{abstract}
The link of the metazoan nucleus to the actin cytoskeleton is highly important for actin polymerization and migration of multiple cell types as well as for mechanotransduction and even affects the cellular transcriptome. Several mechanisms of organization of actin filaments next to the nuclear envelope have been identified. Among these mechanisms the most studied one is the Linker of nucleoskeleton and cytoskeleton (LINC) complex-dependent perinuclear actin organization. However, recently additional mechanisms have been identified: an Actin-related protein-2/3 (Arp2/3)-dependent perinuclear actin polymerization during migration of dendritic cells and a perinuclear actin rim that is formed in response to external force application or migration cues. In parallel, there are also reports on cancer cells that migrate in a LINC complex independent manner and on cancers with reduced expression of the LINC complex components. Thus, suggesting that LINC complex independent migration may be associated with tumour formation.
\end{abstract}

$\begin{array}{ll}\text { Abbreviations } \\ \text { 2/3D: } & \text { Two/three-dimensional } \\ \text { MRTF-A: } & \text { Myocardin related } \\ \text { ARP2/3: } & \text { Actin-related protein-2/3 transcription factor-A } \\ \text { CaAR: } & \text { Calcium-mediated actin reset } \\ \text { NPC: } & \text { Nuclear pore complex } \\ \text { INF2: } & \text { Inverted formin-2 } \\ \text { ONM: } & \text { Outer nuclear membrane } \\ \text { INM: } & \text { Inner nuclear membrane } \\ \text { PNS: } & \text { Perinuclear space } \\ \text { KASH: } & \text { Klarsicht, ANC-1, Syne Homology domain } \\ \text { SUN: } & \text { Sad1p, UNC-84 } \\ \text { LINC: } & \text { Linker of nucleoskeleton and cytoskeleton } \\ \text { TAN: } & \text { Transmembrane actin-associated nuclear }\end{array}$

\section{Introduction}

The nuclear envelope separates the eukaryotic nucleus from the rest of the cell. Apart from generating a physical barrier, the nuclear envelope is actively involved in several processes including organization of nuclear architecture, transcriptional control, and migration of both the nucleus and the cell. The

\footnotetext{
*Address correspondence to: Gabi Gerlitz, gabige@ariel.ac.il Received: 05 July 2021; Accepted: 08 September 2021
}

nuclear envelope is composed of the Nuclear Pore Complexes (NPCs) and the nuclear membrane, encompassing an inner nuclear membrane (INM, facing the inside of the nucleus) and an outer nuclear membrane (ONM, facing the cytoplasm) (Hetzer, 2010; Wilson and Berk, 2010). The ONM can interact with various cytoskeletal elements, including actin filaments, while in metazoans the INM is connected to a filamentous network termed the nuclear lamina (de Leeuw et al., 2018; Gruenbaum and Medalia, 2015). The nuclear lamina is composed of lamins, which are type $\mathrm{V}$ intermediate filament proteins classified into A-type and B-type lamins. They are key components for determining the structure and the mechanostability of the nucleus, but they have many other roles, for example in chromatin organization and DNA damage repair (Donnaloja et al., 2020; Gruenbaum and Foisner, 2015; Ho and Lammerding, 2012; Patil and Sengupta, 2021).

The nuclear envelope is connected to the various cytoskeletal networks in the cytoplasm including actin filaments. Actin association with the nuclear envelope is important for correct positioning of the nucleus in polarized cells, cell migration, mechanotransduction and transcriptional control (Davidson and Cadot, 2021).

\section{The LINC Complex}

The major known nuclear envelope component that facilitates interaction between the nucleus and the cytoskeleton is the 


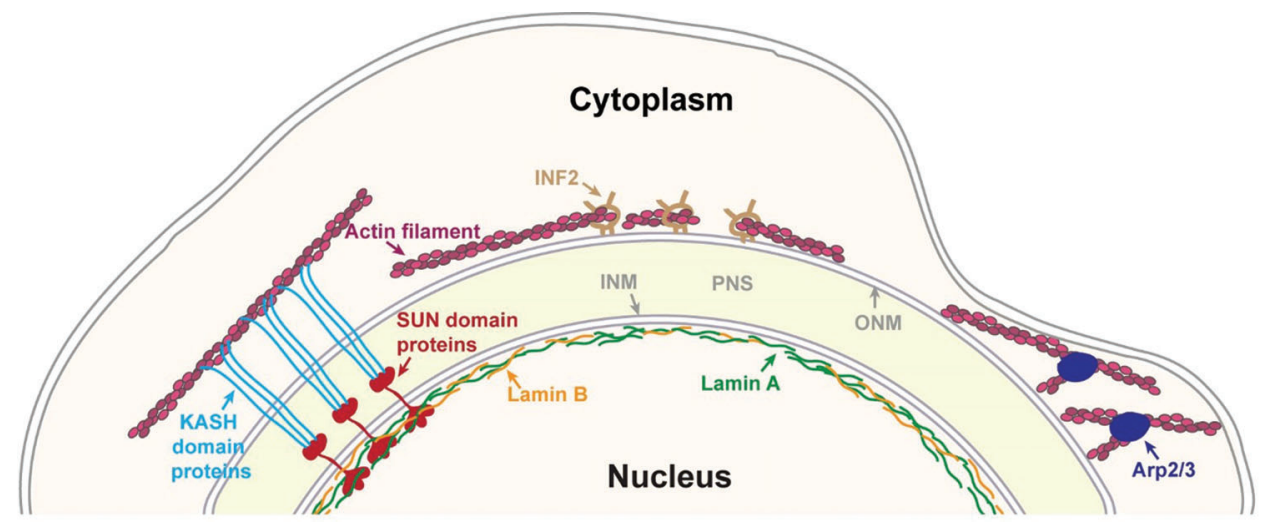

FIGURE 1. Major mechanisms of perinuclear actin organization. The nucleus is separated from the cytoplasm by the nuclear envelope, which is composed by an outer nuclear membrane (ONM) and an inner nuclear membrane (INM) that are separated by the perinuclear space (PNS). The INM is connected to the nuclear lamina that is generated by A and B-type lamins. One mechanism to link actin filaments to the nucleus is by SUN domain and KASH domain proteins that interact inside the PNS to form the Linker of nucleoskeleton and cytoskeleton (LINC) complex. Perinuclear actin filaments can be generated by Inverted formin 2 (INF-2) to form a perinuclear actin rim. Perinuclear actin rim was also found to be sensitive to increased lamin B1 levels. In dendritic cells that pass through small constraints Actin-related protein-2/3 (Arp2/3) promotes the formation of perinuclear actin filaments.

Linker of nucleoskeleton and cytoskeleton (LINC) complex. This complex is formed by Sad1p/UNC-84 (SUN) domain proteins and Klarsicht, ANC-1, Syne Homology (KASH) domain proteins, which cross the INM and the ONM, respectively. SUN domain proteins were shown to form trimers while their C-termini interact with the C-termini of three KASH domain proteins to form stable hexamers. These hexamers may further interact with each other to generate higher order complexes (Hao and Starr, 2019; Jahed et al., 2021). The N-terminus of the SUN domain proteins extends into the nucleus to interact with nuclear factors such as the nuclear lamina, nuclear membrane proteins and chromosomes. The $\mathrm{N}$-terminus of the $\mathrm{KASH}$ domain proteins extends into the cytoplasm to interact with various cytoskeletal elements including actin filaments and microtubule motor proteins (Fig. 1) (Jahed and Mofrad, 2018; Rothballer and Kutay, 2013; StewartHutchinson et al., 2008).

The LINC complex was shown to be crucial for linking several cytoplasmic actin structures to the nucleus including the actin cap and the transmembrane actin-associated nuclear (TAN) lines (Davidson and Cadot, 2021). The actin cap is a structure composed of thick and highly contractile bundles of actomyosin filaments loaded on top of the nucleus in polarized primary cells that functions in maintaining nuclear shape and in pulling the nucleus towards the front of the cell during migration (Khatau et al., 2009; Kim et al., 2014; Maninova et al., 2017). TAN lines are linear arrays of nuclear envelope membrane proteins composed of the LINC proteins Nesprin-2G and SUN2, which are formed on the dorsal surface of the nuclear envelope. TAN lines are formed along actin cables that move towards the rear end of the cell. The link of the actin cables to the nucleus by the LINC complex and lamin A results in rearward nuclear movement during early stages of cell migration to polarize the cell (Kutscheidt et al., 2014; Luxton et al., 2010).

As the major linker of the cytoskeleton to the nucleus, the LINC complex was found to have a key role in multiple processes including mechanotransduction, chromosome organization, DNA repair, cell migration and cytoskeleton organization (Bone and Starr, 2016; Hao and Starr, 2019; Hieda, 2019; Jahed and Mofrad, 2018; Khilan et al., 2021; Osorio and Gomes, 2014; Wong et al., 2021). However, in recent years it appears that in some cases actin filaments may accumulate next to the nucleus by LINC complex independent mechanisms.

\section{Perinuclear Actin Independent of the LINC Complex}

One type of a LINC complex independent perinuclear actin structure is a transient actin polymerization around the nucleus by the Arp2/3 complex. This phenomenon was found in mouse dendritic cells migrating through small constraints in both in vitro and in vivo systems (Fig. 1). Knockdown of SUN1 in SUN2 knockout cells did not interfere with this perinuclear actin accumulation, whereas knockdown of lamin A did lead to reduced accumulation of actin around the nucleus (Thiam et al., 2016). Notably, lamin $\mathrm{A}$ is known to associate with increased nuclear rigidity (Goldberg et al., 2008; Harada et al., 2014; Lammerding et al., 2006; Stephens et al., 2017; Swift et al., 2013; Zhang et al., 2019). In addition, the observation that internalized large beads accumulated similar actin filaments around them during confined cell migration (Thiam et al., 2016) suggests that the trigger for actin polymerization is just the relative high stiffness of the nucleus. It was shown that these perinuclear actin filaments deform the nucleus to enable its passage through small constraints, though it is still not clear how Arp2/3 is activated (Thiam et al., 2016).

Another perinuclear actin structure that is LINCindependent is the perinuclear actin rim (Fracchia et al., 2020; Shao et al., 2015a; Shao et al., 2015b) that was also termed Calcium-mediated actin reset (CaAR) (Wales et al., 2016). A transient formation of perinuclear actin filaments around the cytosolic side of the nuclear envelope of cells in two-dimensional (2D) culture was found in several cell 
types including mouse fibroblasts, breast cancer cells and kidney epithelial cells (Shao et al., 2015a; Shao et al., 2015b; Wales et al., 2016). This actin structure was found to form for a period of 1-5 minutes in response to application of external force that led to calcium ions influx, which in turn activated the actin polymerization promoting factor Inverted formin 2 (INF-2) (Fig. 1) (Shao et al., 2015b; Wales et al., 2016). In mouse fibroblasts inhibition of the LINC complex by overexpression of a dominant negative KASH domain did not interfere with the perinuclear actin rim formation (Shao et al., 2015b). Overexpression of the KASH domain of either Nesprin 1 or Nesprin 2 saturates the endogenous SUN domain proteins, thus it interferes with the formation of the endogenous LINC complex (Lombardi et al., 2011; StewartHutchinson et al., 2008).

We were able to detect a similar perinuclear actin rim in mouse melanoma cells migrating in a 2D system or embedded in a 3D system of collagen fibers that was also LINC complexindependent but had a much longer lifetime, on a time scale of hours. This stable perinuclear actin rim was induced by migration cues and was sensitive to elevated levels of lamin B1, while it was not affected by either higher levels of lamin A or inhibition of the LINC complex by overexpression of a dominant negative KASH domain (Fig. 1) (Fracchia et al., 2020). The ability of lamin B1 that is localized at the inner side of the nuclear envelope, to affect LINC complex independent actin filaments at the outer side of the nuclear envelope suggests an inside-out signal transduction mechanism. It may be based on force transmission due to altered nuclear envelope stiffness upon changes in lamin B1 levels. Alternatively, it may indicate the existence of a protein-based physical linkage between the nuclear lamina and perinuclear actin filaments that is not based on KASH domain and SUN domain proteins.

The role of the perinuclear actin rim is not clear. The transient perinuclear actin rim was suggested to promote cell migration by supporting changes in transcription that occurred due to translocation of the transcription coactivator Myocardin related transcription factor-A (MRTF-A) from the cytoplasm to the nucleus (Wales et al., 2016). On the other hand, we found that the stable perinuclear actin rim in melanoma cells reduced the cellular migration rate (Fracchia et al., 2020). Intensive nuclear envelope stretching during cell migration can disrupt its integrity and lead to DNA damage (Denais et al., 2016; Raab et al., 2016). Thus, we hypothesize that the stable perinuclear actin rim may be involved in protection of chromatin from a mechanical damage during the migration process by increasing the rigidity of the nuclear envelope and limiting nuclear envelope folding.

\section{LINC Complex Independent Cell Migration}

The findings of perinuclear actin structures that are LINC complex independent suggest that there should be cells that migrate without the support of the LINC complex. Indeed, migration of cells in a LINC complex independent manner or even in a faster rate upon inhibition of the LINC complex was reported for mouse melanoma cells (Fracchia et al., 2020), human lung cancer cells (Lv et al., 2015) and rat mammary adenocarcinoma cells (Sharma et al., 2021). Still, it should be noted that there are other cancer cells that do require the LINC complex to support their migration (Colón-Bolea et al., 2020; Imaizumi et al., 2018; Infante et al., 2018). Interestingly, in parallel there are accumulating reports on down-regulation of LINC complex components in various cancer types such as breast cancer, lung cancer, prostate cancer and liver cancer (Cartwright and Karakesisoglou, 2014; Lv et al., 2015; Marmé et al., 2008; Matsumoto et al., 2015; Sharma et al., 2021; Sur-Erdem et al., 2020; Tessema et al., 2008; Yajun et al., 2017). Taken together, these recent data may suggest that some cancer cells acquire alternative mechanisms that are LINC complex independent to connect actin filaments to their nuclei or to disconnect their nucleus from the actin network to enhance their migration capabilities. These mechanisms are still to be discovered.

Author Contribution: The authors confirm contribution to the paper as follows: study conception and design: A.F. \& G.G.; analysis and interpretation of results: A.F. \& G.G.; draft manuscript preparation: A.F. \& G.G. All authors reviewed the results and approved the final version of the manuscript.

Funding Statement: This research was funded by the Israel Cancer Association, Grant No. 20190028 and Ariel University.

Conflicts of Interest: The authors declare that they have no conflicts of interest to report regarding the present study.

\section{References}

Bone CR, Starr DA (2016). Nuclear migration events throughout development. Journal of Cell Science 129: 1951-1961. DOI $10.1242 /$ jcs. 179788.

Cartwright S, Karakesisoglou I (2014). Nesprins in health and disease. Seminars in Cell \& Developmental Biology 29: 169179. DOI 10.1016/j.semcdb.2013.12.010.

Colón-Bolea P, García-Gómez R, Shackleton S, Crespo P, Bustelo XR et al. (2020). RAC1 induces nuclear alterations through the LINC complex to enhance melanoma invasiveness. Molecular Biology of the Cell 31: 2768-2778. DOI 10.1091/ mbc.E20-02-0127.

Davidson PM, Cadot B (2021). Actin on and around the Nucleus. Trends in Cell Biology 31: 211-223. DOI 10.1016/j.tcb.2020.11.009.

de Leeuw R, Gruenbaum Y, Medalia O (2018). Nuclear lamins: Thin filaments with major functions. Trends in Cell Biology 28: 3445. DOI 10.1016/j.tcb.2017.08.004.

Denais CM, Gilbert RM, Isermann P, McGregor AL, te Lindert M et al. (2016). Nuclear envelope rupture and repair during cancer cell migration. Science 352: 353-358. DOI 10.1126/ science.aad7297.

Donnaloja F, Carnevali F, Jacchetti E, Raimondi MT (2020). Lamin A/C Mechanotransduction in Laminopathies. Cells 9: 1306. DOI 10.3390/cells9051306.

Fracchia A, Asraf T, Salmon-Divon M, Gerlitz G (2020). Increased lamin B1 levels promote cell migration by altering perinuclear actin organization. Cells 9: 2161. DOI 10.3390/cells9102161.

Goldberg MW, Huttenlauch I, Hutchison CJ, Stick R (2008). Filaments made from A- and B-type lamins differ in structure and organization. Journal of Cell Science 121: 215-225. DOI $10.1242 /$ jcs.022020. 
Gruenbaum Y, Foisner R (2015). Lamins: Nuclear intermediate filament proteins with fundamental functions in nuclear mechanics and genome regulation. Annual Review of Biochemistry 84: 131-164. DOI 10.1146/annurev-biochem-060614-034115.

Gruenbaum Y, Medalia O (2015). Lamins: The structure and protein complexes. Current Opinion in Cell Biology 32: 7-12. DOI 10.1016/j.ceb.2014.09.009.

Hao H, Starr DA (2019). SUN/KASH interactions facilitate force transmission across the nuclear envelope. Nucleus 10: 7380. DOI 10.1080/19491034.2019.1595313.

Harada T, Swift J, Irianto J, Shin JW, Spinler KR et al. (2014). Nuclear lamin stiffness is a barrier to $3 \mathrm{D}$ migration, but softness can limit survival. Journal of Cell Biology 204: 669-682. DOI 10.1083/jcb.201308029.

Hetzer MW (2010). The Nuclear Envelope. Cold Spring Harbor Perspectives in Biology 2: a000539. DOI 10.1101/cshperspect. a000539.

Hieda M (2019). Signal transduction across the nuclear envelope: Role of the LINC complex in bidirectional signaling. Cells 8: 124 . DOI $10.3390 /$ cells 8020124 .

Ho CY, Lammerding J (2012). Lamins at a glance. Journal of Cell Science 125: 2087-2093. DOI 10.1242/jcs.087288.

Imaizumi H, Sato K, Nishihara A, Minami K, Koizumi M et al. (2018). X-ray-enhanced cancer cell migration requires the linker of nucleoskeleton and cytoskeleton complex. Cancer Science 109: 1158-1165. DOI 10.1111/cas.13545.

Infante E, Castagnino A, Ferrari R, Monteiro P, Agüera-González S et al. (2018). LINC complex-Lis1 interplay controls MT1MMP matrix digest-on-demand response for confined tumor cell migration. Nature Communications 9: 8610. DOI 10.1038/s41467-018-04865-7.

Jahed Z, Domkam N, Ornowski J, Yerima G, Mofrad MRK (2021). Molecular models of LINC complex assembly at the nuclear envelope. Journal of Cell Science 134: 682. DOI 10.1242/ jcs.258194.

Jahed Z, Mofrad MRK (2018). Mechanical LINCs of the nuclear envelope: Where SUN meets KASH. Extreme Mechanics Letters 20: 99-103. DOI 10.1016/j.eml.2018.01.010.

Khatau SB, Hale CM, Stewart-Hutchinson PJ, Patel MS, Stewart CL et al. (2009). A perinuclear actin cap regulates nuclear shape. Proceedings of the National Academy of Sciences of the United States of America 106: 19017-19022. DOI 10.1073/ pnas.0908686106.

Khilan AA, Al-Maslamani NA, Horn HF (2021). Cell stretchers and the LINC complex in mechanotransduction. Archives of Biochemistry and Biophysics 702: 108829. DOI 10.1016/j. abb.2021.108829.

Kim DH, Cho S, Wirtz D (2014). Tight coupling between nucleus and cell migration through the perinuclear actin cap. Journal of Cell Science 125: 1099. DOI 10.1242/jcs.144345.

Kutscheidt S, Zhu R, Antoku S, Luxton GWG, Stagljar I et al. (2014). FHOD1 interaction with nesprin-2G mediates TAN line formation and nuclear movement. Nature Cell Biology 16: 708-715. DOI 10.1038/ncb2981.

Lammerding J, Fong LG, Ji JY, Reue K, Stewart CL et al. (2006). Lamins $\mathrm{A}$ and $\mathrm{C}$ but not lamin $\mathrm{B} 1$ regulate nuclear mechanics. Journal of Biological Chemistry 281: 2576825780. DOI 10.1074/jbc.M513511200.

Lombardi ML, Jaalouk DE, Shanahan CM, Burke B, Roux KJ et al. (2011). The interaction between nesprins and sun proteins at the nuclear envelope is critical for force transmission between the nucleus and cytoskeleton.
Journal of Biological Chemistry 286: 26743-26753. DOI 10.1074/jbc.M111.233700.

Luxton GWG, Gomes ER, Folker ES, Vintinner E, Gundersen GG (2010). Linear arrays of nuclear envelope proteins harness retrograde actin flow for nuclear movement. Science 329: 956-959. DOI 10.1126/science.1189072.

Lv X, Liu L, Cheng C, Yu B, Xiong L et al. (2016). SUN2 exerts tumor suppressor functions by suppressing the Warburg effect in lung cancer. Scientific Reports 5: 5465. DOI 10.1038/srep17940.

Maninova M, Caslavsky J, Vomastek T (2017). The assembly and function of perinuclear actin cap in migrating cells. Protoplasma 254: 1207-1218. DOI 10.1007/s00709-017-1077-0.

Marmé A, Zimmermann HP, Moldenhauer G, Schorpp-Kistner M, Müller C et al. (2008). Loss of Drop1 expression already at early tumor stages in a wide range of human carcinomas. International Journal of Cancer 123: 2048-2056. DOI 10.1002/ijc.23763.

Matsumoto A, Hieda M, Yokoyama Y, Nishioka Y, Yoshidome K et al. (2015). Global loss of a nuclear lamina component, lamin A/C, and LINC complex components SUN1, SUN2, and nesprin-2 in breast cancer. Cancer Medicine 4: 15471557. DOI 10.1002/cam4.495.

Osorio DS, Gomes ER (2014). Connecting the Nucleus to the Cytoskeleton for Nuclear Positioning and Cell Migration. In: Schirmer EC, de las Heras JI (eds.), Cancer Biology and the Nuclear Envelope, vol. 773, pp. 505-520. New York, NY, New York: Springer. DOI 10.1007/978-1-4899-8032-8_23.

Patil S, Sengupta K (2021). Role of A- and B-type lamins in nuclear structure-function relationships. Biology of the Cell 113: 295310. DOI 10.1111/boc. 202000160 .

Raab M, Gentili M, de Belly H, Thiam HR, Vargas P et al. (2016). ESCRT III repairs nuclear envelope ruptures during cell migration to limit DNA damage and cell death. Science 352: 359-362. DOI 10.1126/science.aad7611.

Rothballer A, Kutay U (2013). The diverse functional LINCs of the nuclear envelope to the cytoskeleton and chromatin. Chromosoma 122: 415-429. DOI 10.1007/s00412-013-0417-x.

Shao X, Kawauchi K, Shivashankar GV, Bershadsky AD (2015a). Novel localization of formin mDia2: Importin -mediated delivery to and retention at the cytoplasmic side of the nuclear envelope. Biology Open 4: 1569-1575. DOI 10.1242/bio.013649.

Shao X, Li Q, Mogilner A, Bershadsky AD, Shivashankar GV (2015b). Mechanical stimulation induces formin-dependent assembly of a perinuclear actin rim. Proceedings of the National Academy of Sciences of the United States of America 112: E2595-E2601. DOI 10.1073/pnas.1504837112.

Sharma VP, Williams J, Leung E, Sanders J, Eddy R et al. (2021). SUN-MKL1 crosstalk regulates nuclear deformation and fast motility of breast carcinoma cells in Fibrillar ECM microenvironment. Cells 10: 1549. DOI 10.3390/ cells10061549.

Stephens AD, Banigan EJ, Adam SA, Goldman RD, Marko JF (2017). Chromatin and lamin A determine two different mechanical response regimes of the cell nucleus. Molecular Biology of the Cell 28: 1984-1996. DOI 10.1091/mbc.e16-09-0653.

Stewart-Hutchinson PJ, Hale CM, Wirtz D, Hodzic D (2008). Structural requirements for the assembly of LINC complexes and their function in cellular mechanical stiffness. Experimental Cell Research 314: 1892-1905. DOI 10.1016/j.yexcr.2008.02.022.

Sur-Erdem I, Hussain MS, Asif M, Pınarbası N, Aksu AC et al. (2020). Nesprin-1 impact on tumorigenic cell phenotypes. 
Molecular Biology Reports 47: 921-934. DOI 10.1007/s11033019-05184-w.

Swift J, Ivanovska IL, Buxboim A, Harada T, Dingal PCDP et al. (2013). Nuclear lamin-A scales with tissue stiffness and enhances matrix-directed differentiation. Science 341: 1240104. DOI 10.1126/science.1240104.

Tessema M, Willink R, Do K, Yu YY, Yu W et al. (2008). Promoter methylation of genes in and around the candidate lung cancer susceptibility locus 6q23-25. Cancer Research 68: 1707-1714. DOI 10.1158/00085472.CAN-07-6325.

Thiam H-R, Vargas P, Carpi N, Crespo CL, Raab M et al. (2016). Perinuclear Arp2/3-driven actin polymerization enables nuclear deformation to facilitate cell migration through complex environments. Nature Communications 7: 501. DOI 10.1038/ncomms10997.
Wales P, Schuberth CE, Aufschnaiter R, Fels J, García-Aguilar I et al. (2016). Calcium-mediated actin reset (CaAR) mediates acute cell adaptations. eLife 5: 990. DOI 10.7554/eLife.19850.

Wilson KL, Berk JM (2010). The nuclear envelope at a glance. Journal of Cell Science 123: 1973-1978. DOI 10.1242/jcs.019042.

Wong X, Loo TH, Stewart CL (2021). LINC complex regulation of genome organization and function. Current Opinion in Genetics \& Development 67: 130-141. DOI 10.1016/j.gde.2020.12.007.

Yajun C, Chen Y, Xiaosa L, Xiao W, Jia C et al. (2017). Loss of Sun2 promotes the progression of prostate cancer by regulating fatty acid oxidation. Oncotarget 8: 89620-89630. DOI 10.18632/oncotarget.19210.

Zhang Q, Tamashunas AC, Agrawal A, Torbati M, Katiyar A et al. (2019). Local, transient tensile stress on the nuclear membrane causes membrane rupture. Molecular Biology of the Cell 30: 899-906. DOI 10.1091/mbc.E18-09-0604. 